\title{
Féeries
}

Études sur le conte merveilleux, XVII ${ }^{\mathrm{e}} \mathrm{XIX}{ }^{\mathrm{e}}$ siècle

16 | 2020

Le conte, les mythes antiques, la Bible

\section{La faille de l'Invincible, de Samson à Kachtcheï}

The failing invincibility, from Samson to Koschei

\section{Pierre-Emmanuel Moog}

\section{(2) OpenEdition}

Journals

Édition électronique

URL : https://journals.openedition.org/feeries/2186

DOI : 10.4000/feeries. 2186

ISSN : 1957-7753

\section{Éditeur}

UGA Éditions/Université Grenoble Alpes

\section{Édition imprimée}

ISBN : 978-2-37747-194-2

ISSN : $1766-2842$

\section{Référence électronique}

Pierre-Emmanuel Moog, "La faille de l'Invincible, de Samson à Kachtcheï », Féeries [En ligne], 16 |

2020, mis en ligne le 07 janvier 2021, consulté le 10 décembre 2021. URL : http://

journals.openedition.org/feeries/2186; DOI : https://doi.org/10.4000/feeries.2186

Ce document a été généré automatiquement le 10 décembre 2021.

(c) Féeries 


\title{
La faille de l'Invincible, de Samson à Kachtcheï
}

\author{
The failing invincibility, from Samson to Koschei
}

Pierre-Emmanuel Moog

1 Les humains ont souvent déploré être parmi les créatures les plus faibles et les plus vulnérables ${ }^{1}$. Il n'est donc pas étonnant qu'ils rêvent d'invincibilité. De nombreux récits ont pris en charge ce fantasme, à commencer par Samson, dans la Bible hébraïque, ou Achille, dans la littérature mythologique gréco-latine. En dehors de ces personnages antiques, complexes et plutôt positifs, par la suite, un personnage a priori invincible figurera dans de nombreux contes, généralement comme antagoniste (sous la catégorie générique de Corps-sans-Âme, ATU 302). Dans tous ces cas, le prétendu personnage invincible est finalement vaincu. L'étude de quelques récits, depuis la Bible $e^{2}$ jusqu'aux Mille et Une Nuits, aux contes des Grimm et d'Afanassiev, et de quelques contes de la tradition orale (de Guyenne et de l'Ariège) nous amènera à repérer un schéma commun à ces récits où la révélation du secret s'avère le point crucial.

2 Lorsque Samson naît, les Hébreux vivent sous le joug des Philistins depuis longtemps. En grandissant, il apparaît doté d'une force invincible et deviendra leur libérateur, jusqu'à sa propre chute. La faillite du dernier des suffètes du Livre des Juges (rédigé vers le VIII ${ }^{e}$ siècle avant l'ère commune) conduira le peuple hébreu à l'anarchie violente (Jg 17-21) avant une difficile reprise en main par le prophète Samuel (1 Sam). Elle n'est donc pas un détail de cette histoire. Comment en est-on arrivé là ? L'idée commune est que Samson perd sa force lorsque sa concubine Dalila lui rase ses cheveux et qu'il lui aurait révélé son secret par bêtise ou par faiblesse psychologique devant l'irrésistible pouvoir de séduction féminin ${ }^{3}$. Pourtant le texte montre bien que Samson, qui vient de raconter par trois fois des balivernes aux interrogations de Dalila, ne peut pas être dupe de ses intentions, puisqu'à chaque fois une tentative de capture par les Philistins s'ensuit (Jg 16,6-14) :

Dalila dit à Samson: «Apprends-moi donc pourquoi ta force est si grande, et avec quoi il faudrait te lier pour te dompter. » Samson lui dit: « Si on me liait avec sept cordelettes fraîches et encore humides, je perdrais ma force et deviendrais 
semblable à un autre homme. » Les princes philistins lui procurent sept cordelettes humides avec lesquelles elle le lie. En embuscade des hommes attendent dans la chambre ; puis elle lui dit : «Les Philistins viennent sur toi, Samson! » Et il rompt ses liens, comme se rompent des liens d'étoupe à l'approche du feu. Et le secret de sa force reste inconnu. Dalila dit à Samson: «Tu t'es joué de moi, tu m'as dit des tromperies! Voyons, fais-moi savoir avec quoi l'on pourrait te lier.» Il lui dit : « Si l'on m'attachait fortement avec des cordes neuves n'ayant pas encore servi, je perdrais ma force et deviendrais comme un autre homme. » Alors Dalila prend des cordes neuves avec lesquelles elle le lie. Elle lui dit : « Les Philistins viennent sur toi, Samson!» En embuscade des hommes attendent dans la chambre. Il arrache les cordes de ses bras comme du fil. Dalila dit à Samson: "Jusque-là, tu t'es raillé de moi et ne m'as dit que des tromperies. Apprends-moi comment on pourrait te lier ! » Il lui dit: «Tu n'aurais qu'à entre-tisser les sept tresses de ma tête avec un ourdissoir. » Lorsqu'elle eut fixé la cheville, elle lui dit : « Les Philistins viennent sur toi, Samson!» Il se réveille de son sommeil et arrache la cheville du métier et l'ourdissoir".

3 En outre, si Samson croyait vraiment révéler le secret de sa force, pourquoi, après s'être laissé raser les cheveux, en se réveillant, tenterait-il pourtant de s'opposer aux Philistins qui se saisissent de lui ( Je m'en sortirai comme de fois en fois, je me secouerai », Jg 16,20) ? Et pourquoi, plus tard en captivité, alors que ses cheveux ont repoussé, croit-il nécessaire d'implorer Dieu de lui donner pour une ultime fois de la force («Seigneur, souviens-toi donc de moi, fortifie-moi donc, rien que cette fois », Jg $16,28)$ ? Une lecture attentive du cycle montre ${ }^{5}$ qu'il faut questionner le lien entre la longue chevelure tressée de Samson et sa force, mais laissons pour le moment la complexité du texte biblique, et examinons ce qu'il en est dans quelques contes.

Dans « La boule de cristal » des Grimm (KHM n 197, $6^{\mathrm{e} e ́ d i t i o n ~ d e ~ 1850), ~ u n ~ h e ́ r o s ~ d e ́ c i d e ~}$ de délivrer une princesse retenue captive par un magicien. Il parvient jusqu'à elle, et l'interroge :

- Comment peux-tu être délivrée ? [...] Je ne crains aucun danger.

- Celui qui parviendra à obtenir la boule de cristal et qui la tient devant le magicien brisera son pouvoir [...] nombreux sont ceux qui ont déjà trouvé la mort ainsi, et j'ai pitié de toi, qui a le sang jeune, à l'idée de savoir que tu affronteras ces grands dangers.

- Rien ne pourra me retenir, mais dis-moi ce que je dois faire [...].

- Je vais tout te dire. En descendant de la montagne sur laquelle se dresse [c]e château, tu trouveras en bas, près d'une source, un auroch sauvage qu'il te faudra combattre. Et si tu parviens à le tuer, il s'élèvera de son cadavre un oiseau de feu qui porte dans son corps un œuf incandescent. Le jaune de cet œuf, c'est la boule de cristal. L'oiseau n'abandonnera pas l'œuf à moins d'y être contraint, mais si cet œuf tombe par terre, il s'enflammera et brûlera tout alentour. Alors l'œuf fondra et la boule de cristal avec lui, si bien que tous tes efforts auront été vains ${ }^{6}$.

Par la suite, bien sûr, le héros réalise l'exploit nécessaire. Mais comment, en premier lieu, la princesse connaît-elle les détails d'une procédure aussi compliquée ? Le texte ne le dit pas, il montre seulement qu'elle ne révèle les instructions pour la sauver qu'avec réticence, car, peut-on supposer, elle estime la tâche irréalisable. De fait, vingt-trois prétendants ont « déjà péri d'une mort misérable [et] un seul pouvait encore essayer de la délivrer, après quoi il ne pourrait plus venir personne ${ }^{7}$ »; or notre héros n'y parviendra que parce qu'il bénéficie de différentes aides magiques. Alors le mystère demeure sur l'origine de la connaissance d'un tel secret par la princesse ${ }^{8}$ : aurait-elle surpris le magicien en train de le répéter lors d'une cérémonie rituelle ? Le lui aurait-il dit lui-même à la manière de Samson? 
6 Dans « L'Ours noir », conte populaire de Guyenne, on en apprend un peu plus. Un héros veut délivrer une princesse cette fois « enlevée par un géant qui l'a emmenée dans une tour au milieu de la mer ${ }^{9}$. Il parvient jusqu'à elle mais la princesse ne connaît apparemment pas les moyens de sa délivrance, puisque, en complicité avec le héros, «le soir quand le géant est rentré, la princesse lui demande d'où viennent "ses forces" 10 ». On peut donc comprendre que la princesse avait admis jusque-là que la force du géant lui était un attribut immuable, et qu'il fallait la finesse du héros pour la convaincre de le lui demander. Visiblement, le géant le lui révèle, la voix narrative indiquant simplement que : «Ses forces sont dans un œuf, qui est dans un pigeon, qui est lui-même dans l'Ours noir de la Forêt Noire. Si quelqu'un lui cassait cet œuf sur le front, le géant serait perdu ${ }^{11}$. » Le style littéraire propre au résumé excluant le discours direct et synthétisant l'information, l'instance narrative est imprécise et les motivations sont nécessairement très réduites. Par la suite, bien sûr, le héros réalise l'exploit nécessaire. Dans ce récit (contrairement à «La boule de cristal»), nous apprenons directement que c'est le ravisseur lui-même qui révèle le secret de sa vulnérabilité à sa captive, mais pourquoi le fait-il donc? Certes, ils se trouvent sur un îlot isolé, et le géant ne sait pas qu'un adversaire, qui plus est valeureux, s'est introduit dans la place. Mais, néanmoins, la princesse étant certainement désireuse de s'enfuir, pourquoi prendre le risque de l'informer ainsi ?

7 Remarquons que les « forces » (ou l'âme, ou la vie, ou la « mort ») de l'antagoniste sont, dans nos histoires, particulièrement peu accessibles: d'abord en étant externes au corps, ensuite en étant abritées derrière une multitude d'enveloppes protectrices. L'externalisation, ou la répartition de ses forces, est l'une des stratégies défensives de base. Quant aux protections pour atteindre l'âme, elles reposent sur l'association d'environnements très différents et en principe incompatibles : le ciel (le pigeon) et la forêt (l'ours noir). On retrouve encore l'idée de l'œuf et de sa coquille, éléments qui combinent les idées de résistance et de fragilité ${ }^{12}$, et, par sa forme régulière sans aspérité, l'idée de la fonction protectrice par excellence ${ }^{13}$, l'œuf représentant donc la genèse et la protection de la vie tout à la fois ${ }^{14}$.

8 Dans «Corps-sans-âme ", conte populaire de l'Ariège, la raison pour laquelle le secret de la vulnérabilité est révélé est formulée plus explicitement. La princesse dit au héros venu la sauver : «[...] le tuer est chose impossible. Il m'en a fait la confidence, cet être a bien une âme, mais elle est séparée de son corps $^{15}$." Ainsi, on peut supposer que l'exploit pour s'emparer de l'âme ennemie apparaît suffisamment impossible à réaliser pour que l'antagoniste prenne le risque de renseigner la princesse (à qui il veut plaire ce faisant, comme l'implique le mot " confidence ", soit le fait de confier à quelqu'un un secret, des pensées ou des sentiments intimes), et qu'elle-même ait intégré le caractère irrémédiable de sa captivité.

9 Dans «La Belle des Belles » d'Afanassiev, Ivan veut délivrer la princesse capturée par Kachtchéi. Il parvient jusqu'à elle, profitant d'une absence du geôlier, mais, «à sa vue, [elle] éclate en sanglots. [...] [II] la presse : "Je t'en prie, demande à Kachtchéï où est sa mort. -Comme tu veux ${ }^{16}$." ". Visiblement, elle aussi est convaincue que la tâche est vaine, et ne se résout qu'avec un sentiment d'impuissance à essayer. Or cette fois ce récit, contrairement à ceux examinés plus haut, nous raconte l'épisode de la révélation du secret d'invincibilité.

10 Le premier soir, Kachtchéï, à son retour, sent l'odeur d'Ivan, mais la princesse parvient à endormir ses soupçons. Durant le souper, elle lui demande sans détour : «Dis-moi, 
Kachtchéi l'Immortel, où est ta mort? [Ce à quoi il répond :] Qu'est-ce que tu en as à faire, femme stupide ? Ma mort est au bout du balai ${ }^{17}$ !» Menterie sarcastique qui ne suffit pas à décourager le couple complice: Ivan, le lendemain durant l'absence de Kachtchéï, « prend le balai et le couvre d'une couche d'or pur ${ }^{18}$ ".

Le deuxième soir, au retour de Kachtchéï (qui sent encore l'odeur d'Ivan mais se laisse duper),

La Belle des Belles prend la chaise, le fait asseoir sur le banc. Contre la porte, il aperçoit le balai doré. «Qu'est-ce que c'est? - Tu vois, Kachtchéï l'Immortel, quel cas je fais de toi, quel soin je prends de ta mort ! - Femme stupide ! Je plaisantais, ma mort est cachée dans la palissade de chêne ${ }^{19}$ !

Nouvelle menterie. Le prétendu désir de prendre soin de lui ne suffit pas, semble-t-il, à le faire parler. Mais le lendemain, nonobstant, Ivan dore la palissade.

Le troisième soir, au retour de Kachtchéï (qui sent encore l'odeur d'Ivan et se laisse duper),

Cette fois la Belle des Belles s'assied sur le banc, lui laisse la chaise. Le regard de Kachtchéi tombe sur la fenêtre : il avise la palissade dorée qui brille comme le feu. «Qu'est-ce que c'est? - Tu vois, Kachtchéi l'Immortel, quel cas je fais de toi, quel soin je prends de ta mort ! Ces paroles furent du goût de Kachtchéi l'Immortel, il dit à la Belle des Belles: "Femme stupide! Je me moquais! Ma mort est dans un œuf, l'œuf dans une cane, la cane dans une souche, et cette souche-là flotte sur l'onde bleue ! » ${ }^{20}$

Qu'est-ce qui fait que, la troisième fois, Kachtchéï révèle enfin la véritable localisation de sa « mort » ? Remarquons d'abord que la princesse apparaît plutôt finaude. Elle n'est pas dupe des balivernes peu crédibles inventées par Kachtchéi et sans doute s'attendait-elle à ce qu'il ne livre pas son secret si facilement. Elle n'insiste pas et ne lui repose pas la question initiale : c'est en se faisant passer pour naïve qu'elle incite son geôlier à continuer à s'amuser d'elle, jusqu'à lui dire la vérité. Sans doute le fait-il mû par plusieurs raisons. Justement, en premier lieu, parce qu'il sous-estime la Belle des Belles, qu'il traite de "femme stupide", alors que c'est lui qui se laisse duper lorsqu'elle endort ses soupçons quant à l'odeur d'Ivan ${ }^{21}$, et qui de ce fait ne réalise pas qu'elle est désormais soutenue par un vaillant sauveur. Ensuite, parce que la princesse rajoute habilement, à son discours doucereux sur ses dorures attentionnées, des marques de respect implicites qui semblent le toucher : la chaise plutôt que le banc, la qualification flatteuse d'immortel (il est significatif de noter que la princesse s'adresse toujours à lui en ajoutant cet épithète, alors qu'elle n'utilise que son nom quand elle parle de lui à Ivan). Cependant, on peut penser que la raison essentielle qui fait prendre à Kachtchéi le risque de dire son secret est sa conviction de l'absolue sureté des protections entourant sa «mort». Or, élaborer des élucubrations génère un effort cognitif psychiquement éprouvant. Une fois le plaisir de la plaisanterie puis de la moquerie passé, Kachtchéi se lasse, alors pourquoi continuer à s'efforcer de cacher la vérité ?

Un autre conte, mettant en scène un personnage a priori invincible, détaille l'épisode de la révélation de sa vulnérabilité : celui du prince Sayf al-Muluk dans Les Mille et Une Nuits. Le prince (parti d'Égypte) finit, après un long périple le portant jusqu'à Canton, par échouer sur une île isolée (du côté de Zanzibar) où il erre pendant des mois avant de parvenir à un château dans lequel il trouve une jeune femme, "portant des vêtements royaux ${ }^{22}$ ", " dans une vaste salle avec des tapis de soie [sur] un lit d'apparat [au pied duquel sont] quarante plateaux présentant, dans des assiettes d'or ou d'argent, 
toutes sortes de mets ${ }^{23} »$. Elle lui raconte son histoire. Dawlat Khatun est une princesse ceylanaise qui, «avec [s]es servantes, [s'étant] déshabill[ées] pour descendre folâtrer dans l'eau [d'un bassin, fut] emportée entre terre et ciel, tandis qu'une voix [lui] disait : "N’aie pas peur, Dawlat Khatun, aie le cœur en paix !" ${ }^{24}$ ». Elle est déposée en ce château où son ravisseur se « [trans]forme [en] jeune homme, fort beau, élégamment vêtu ${ }^{25}$ ». Il est le fils du roi des djinns, et lui explique qu'étant tombé amoureux d'elle, il l'a « emmen[é] en ce château de belle pierre où [il] habite et où personne ne vient, homme ou génie ${ }^{26}$ ». Il précise : « Tu dois te persuader que tu ne reverras plus les pays de l'Inde ni ta mère. Tu vas rester ici avec moi, le cœur et l'esprit tranquilles, et je te donnerai tout ce que tu me demanderas ${ }^{27}$ ", puis "il [1]'étreignît et [lui] donna des baisers ${ }^{28}$ " mais sans abuser sexuellement d'elle (Dawlat Khatun explique à Sayf al-Muluk qu'il ne lui rend visite qu'un seul jour par semaine pour le passer «en [s]a compagnie [mais qu'elle est toujours] vierge [et que] le djinn [1]'a respectée $\left.{ }^{29} »\right)$. Le prince propose de s'enfuir mais la princesse répond : «Grand Dieu, nous ne le pouvons pas! [...] Ce maudit nous rattraperait et nous tuerait [car] tu ne pourras le mettre à mort que si tu tues son $a^{a m} e^{30}$. » Naturellement, le prince lui demande aussitôt : «Et où loge-t-elle son âme ${ }^{31}$ ? » C'est alors que la princesse explique les circonstances dans lesquelles le djinn le lui a révélé :

Je lui ai posé maintes fois la question, mais il ne m'a rien dit. Une fois pourtant, comme je le pressais, il s'est mis en colère et m'a demandé pourquoi je l'assaillais de questions sur son âme; je lui ai répondu « Ô Hatîm, je n'ai maintenant personne en dehors de toi et de Dieu. Aussi longtemps que je vivrai, je suis liée à ton âme : si je ne la garde pas précieusement au creux de mes yeux, comment pourrais-je vivre quand tu me quittes? Si je savais tout sur cette âme, je la garderais aussi précieusement que mon œil droit. » Il m'a dit alors : « Les astronomes ont prédit à ma naissance que la mort de mon âme ne pouvait venir que d'un homme fils de roi. Alors, je l'ai prise et déposée dans l'estomac d'un moineau que j'ai enfermé dans une boîte, puis la boîte dans une cassette, celle-ci dans une autre, et ainsi de suite jusqu'à sept, la dernière dans une caisse, celle-ci dans une autre et de nouveau jusqu'à sept, la dernière enfin dans un coffre de marbre que j'ai enfoui, près du rivage, dans l'Océan inaccessible, de ce côté, à tout être humain. Voilà ce que j'avais à te dire, mais ne va le répéter à personne. C'est un secret entre toi et moi. » [...] Je lui dis alors : «Et à qui pourrais-je parler de ça ? Il ne vient ici personne d'autre que toi ! Par Dieu, tu as confié ton âme à une forteresse imprenable, exceptionnelle, inaccessible à tous! Quel être humain pourrait réussir l'impossible, à moins que Dieu n'ait décidé de donner suite à la prédiction des astronomes ? Oui, quel être humain pourrait y réussir? » Hatîm alors m'a répondu : «Il faudrait que ce fût un homme qui eût à son doigt l'anneau de Salomon, fils de David - sur eux le salut ! Il poserait la main où est l'anneau à la surface de la mer, invoquerait les noms qui y sont gravés et ordonnerait à la vie cachée dans le coffre d'apparaître. Le coffre apparaitrait, l'homme le briserait avec tout le reste, jusqu'à la boîte où se trouve le moineau : il n'aurait plus qu'à s'en saisir et moi, je mourrais. " $^{32}$

D'évidence, les raisons qui font que le djinn finit lui aussi par livrer son secret diffèrent en partie de celles de Kachtchéï. Lui ressent une affection réelle pour sa captive, qui n'est d'ailleurs pas enfermée (le prince Sayf parvient sans difficulté jusqu'à elle), en dehors de son isolement insulaire. Il la traite avec tendresse, respect et générosité. Il croit naïvement à la bienveillance de la princesse qui s'adresse à lui de manière caressante ( Ô Hatîm» lui dit-elle, se référant sinon à lui successivement par «le génie ", «le djinn", «ce maudit», «Hatîm», «le djinn", «ce maudit», «ce misérable $\aleph^{33}$ ) et feint d'avoir accepté sa présence définitive en ces lieux. Elle reconnaîtrait désormais qu'elle est «liée à [s]on âme [et souhaiterait la] garde[r] 
précieusement ${ }^{34}$ »! Habilement, elle utilise même les longues absences régulières du djinn pour justifier son besoin de se protéger, usant d'un chantage affectif. En fait, le djinn cherche, en lui disant tout, à installer un climat de confiance réciproque, et, si ce n'est une affection mutuelle, au moins une intimité partagée.

On remarque cependant que le djinn, initialement, résistait à divulguer son secret, et ne s'est ouvert que parce que la princesse «le pressai[t de sorte qu']il s'est mis en colère et [lui] a demandé pourquoi [elle] l'assaillai[t] de questions ${ }^{35}$ ». Cet emportement manifeste la tension interne du djinn qui veut à la fois protéger son secret et établir un dialogue sincère et entrer en confidence. Cependant, le djinn ne livre d'abord qu'une partie de l'explication, et doit être relancé pour en donner la totalité, car il sait qu'une infime possibilité demeure d'être vaincu. Mais il n'en reste pas moins que la raison principale des révélations du djinn ressortit à «l'impossib[ilité] » apparente qu'il y aurait à atteindre son âme si bien cachée et protégée, comme pour ses homologues antagonistes des autres récits (respectivement le géant, Corps-sans-âme, Kachtchéï).

On peut d'ailleurs les comprendre, car ce n'est en effet que grâce à un concours de circonstances extraordinaires (et mêmes merveilleuses) que les héros, désirant sauver les princesses, réussissent à réaliser de tels exploits. Celui de "La boule de cristal » bénéficie de l'aide de ses frères, changés par sortilège l'un en aigle et l'autre en baleine. Ceux de "L'Ours noir " et de "Corps-sans-âme » avaient reçu antérieurement, en récompense de leur aide à des animaux, le pouvoir de se changer en ours et en oiseau pour le premier, et en lion, en aigle et en fourmi pour le second. Ivan, à la recherche de la mort de Kachtchéï, se montre en chemin compatissant envers des animaux qui lui offrent leur aide dans sa quête. Enfin, il se trouve que Sayf était justement en possession du fameux et indispensable "anneau de Salomon »: il avait été offert par Salomon lui-même au roi son père ${ }^{36}$ et celui-ci l'avait proposé à Sayf parmi d'autres cadeaux dont un sabre, mais c'est justement l'anneau que Sayf avait choisi ${ }^{37}$. Les différents antagonistes ne pouvaient pas s'imaginer que de telles conditions puissent être réunies, et ils ont présumé de l'impossibilité de détruire leur force, en prenant le risque d'en révéler le secret. En somme, leur vulnérabilité réside précisément dans leur trop grande confiance en leur invincibilité. Ce mécanisme narratif pourrait-il maintenant nous aider à comprendre le cas de Samson?

Nous avons vu qu'en apparence Samson semble révéler le secret de sa force (sa longue chevelure), mais que cette lecture traditionnelle ne résiste pas à une lecture attentive des éléments du texte. Remarquons déjà que dans tous les autres récits examinés, le protagoniste ne livre son secret que parce qu'il est persuadé, à tort, que la tâche qu'il indique est impossible à réaliser : mais quoi de plus facile que de couper des cheveux ?

En fait, si la confidence de Samson se situe vers la fin du cycle, d'autres épisodes méritent aussi examen. Ainsi, au début de son parcours, alors qu'il vient d'affronter un lion avec succès, en le terrassant à mains nues («il le dépèce comme se dépèce un chevreau, et rien, il n'avait rien en ses mains », Jg 14,6b), curieusement il préfère taire cet exploit à ses proches ( «il ne rapporte pas à son père, ni à sa mère, ce qu'il avait fait ", Jg 14,6c ${ }^{38}$; d'autre part, au milieu du cycle, après vingt ans de pouvoir, pourquoi le suffète Samson s'aventure-t-il en territoire ennemi, à Gaza, pour se rendre auprès d'une prostituée puis soudainement à minuit s'emparer des portes de la ville (Jg 16,1-3) $)^{39}$ ? Il apparaît que, dans le cycle biblique de Samson, pour comprendre l'itinéraire du personnage, il faut distinguer trois niveaux: la nature de sa force, l'origine de sa force et la finalité de sa force. Lorsque Samson est encore jeune homme, 
la rencontre avec le lion va lui révéler à lui-même, comme au lecteur, le caractère surnaturel de sa force. Mais le garçon aura cependant d'abord du mal à le croire luimême et n'osera en parler à personne. Aussi, son entourage, Hébreux comme Philistins, ne finira par intégrer cette réalité que beaucoup plus tard, et les Philistins cesseront alors de tenter de s'emparer de lui par la force. À partir du moment où l'on parvient à cette étape, naturellement l'on s'interroge sur l'origine de cette force. Si le lecteur sait, lui, dès le début, qu'elle est de source divine, Samson lui-même passera une partie de sa vie à se demander si sa force provient de sa chevelure, de son statut de nazir, de Dieu, ou encore lui serait intrinsèque. Quant aux Philistins, ayant accepté définitivement, après l'épisode des portes de Gaza, qu'il est vain de tenter de s'opposer à Samson par une force supérieure, ils visent désormais à en découvrir le secret en soudoyant Dalila.

Samson sait bien que, étant donné sa chevelure exceptionnelle, sa force semble être liée à cette particularité. Aussi, il s'amuse d'abord à inventer des balivernes crédibles qui s'approchent de plus en plus de son obligation, en tant que nazir, de ne pas se raser les cheveux. Il est sans doute désormais convaincu d'être naturellement invincible. C'est pourquoi il se débat, croyant pouvoir échapper à ses assaillants, même après qu'on lui a coupé les cheveux. Alors, cependant, si sa force ne provient pas de ses cheveux, pourquoi la perd-il de manière concomitante à son rasage ? En indiquant à Dalila que ses cheveux sont la clé, il sait pertinemment qu'on va les lui couper, ce qui est une rupture de son statut de nazir, et donc du serment passé par sa mère que son fils serait consacré à la délivrance d'Israël : Dieu n'a donc plus de raison de lui insuffler sa force surnaturelle. Samson, désormais en captivité, aura le temps de réfléchir à sa vie et à l'origine divine de sa force, ce qui l'amènera enfin à s'interroger sur sa finalité. Entretemps, sa prétention à se croire lui-même intrinsèquement invincible, et donc à avoir imaginé pouvoir rompre son engagement de nazir impunément, aura été finalement la véritable cause de sa chute.

Cette revue de personnages quasi invincibles nous a emmenés à travers des récits d'époques très différentes, d'aires culturelles variées, et de longueurs plutôt courtes, mais inégalement. Certes, le cycle de Samson développe une complexité plus grande que les autres récits, dans la mesure où il aborde non seulement l'origine et la perte de la force, mais aussi la découverte de sa (sur)nature et de son intensité, et surtout sa finalité (alors que l'existence d'une quasi-invincibilité est une donnée dans les autres récits, puisqu'elle se rapporte à des personnages antagonistes et non au protagoniste principal), en raison de sa visée théologique.

Pourtant, concernant la perte de la force, ces récits reprennent le même schéma: le secret de sa force n'est pas subtilisé à son insu à son détenteur, mais c'est lui-même qui finit par (trop) parler, livrant une vraie ou fausse révélation. Ses motifs sont variés : pour se confier ou pour se vanter, par dédain ou par chagrin, et souvent aussi de guerre lasse, persuadé qu'en fin de compte sa force est soit inaccessible, soit réside intrinsèquement en lui. Or il se trouve qu'étant donné les faits à leur disposition, ces personnages ont de bonnes raisons de croire les choses ainsi. Mais ils ne maitrisent pas tout, et notamment la combinaison improbable (et malheureuse pour eux) des coïncidences et des qualités insoupçonnées et insoupçonnables de leurs adversaires. Peut-on alors imaginer qu'un mécanisme, transcendant ou immanent, soit ici à l'œuvre? Ces êtres dotés d'une surnature ayant émergé à des fins mystérieuses, une justice supérieure, jugeant qu'ils seraient atteints d'une sorte d'hubris et en feraient mauvais usage, favoriserait-elle la conjonction des conditions de leur déchéance? Le 
message que délivrent ces récits est en tout cas essentiellement le même : la conviction qu'ont les puissants qu'ils prévaudront toujours, sans avoir égard à l'usage avisé ou non de leur puissance, et qu'en cela, précisément, peut résider leur ultime faiblesse.

\section{BIBLIOGRAPHIE}

\section{Bibliographie}

\section{Textes}

AfANASSIEV Alexandre, Les Contes populaires russes, trad. L. Gruel-Apert, Paris, Maisonneuve et Larose, 2000.

BRU Josiane, Le Conte populaire français. Contes merveilleux. Supplément au Catalogue de Paul Delarue et Marie-Louise Tenèze, Toulouse, PUM, 2017.

La Bible [1899], trad. du Rabbinat, Z. Kahn (dir.), Paris, Colbo, 1966.

La Bible, trad. A. Chouraqui, Paris, Desclée de Brouwer, 1989.

DelARUE Paul \& TENÈZE Marie-Louise, Le Conte populaire français, Paris, Maisonneuve et Larose, 2002.

GRIMM Jacob \& Wilhelm, Contes pour les enfants et la maison, trad. N. Rimasson-Fertin, Paris, José Corti, 2009, vol. 1.

LA FONTAINE Jean (de), Fables, contes et nouvelles, éd. E. Pilon et R. Groos, Paris, Gallimard, coll. «Bibliothèque de la Pléiade ", 1948.

PASCAL Blaise, Pensées, éd. F. Kaplan, Paris, Cerf, 1982.

Perrault Charles, Contes, éd. T. Gheeraert, Paris, Honoré Champion, 2012.

Platon, Protagoras, Euthydème, Gorgias, Ménexène, Ménon, Cratyle, trad. É. Chambry, Paris, GF Flammarion, 1992.

STACE, Achiléide, trad. J. Méheust, Paris, Les Belles Lettres, 1971.

ToLKIEN J. R. R., Le Hobbit [1937], trad. D. Lauzon, Paris, Christian Bourgois, 2012.

Les Mille et Une Nuits [1991], trad. J. E. Bencheikh et A. Miquel, Paris, Gallimard, coll. «Bibliothèque de la Pléiade ", 2006, t. III.

\section{Études}

ALTER Robert, Ancient Israel: The Former Prophets: Joshua, Judges, Samuel, and Kings: A Translation with Commentary, New York, Norton, 2013. 
Assis Elie, «The Structure and Meaning of the Samson Narratives », dans E. Eynikel et T. Nicklas (éds), Samson: Hero or Fool? The Many Faces of Samson, Leiden, Brill, 2014.

GreENSTEIN Edward, « The Riddle of Samson », Prooftexts, vol. 1, n 3, 1981, p. 237-260.

LUCIANI Didier, « Samson : l'amour rend aveugle », Vetus Testamentum, vol. 59, n², 2009, p. 323-326.

Moog Pierre-Emmanuel, « Samson, de la rumeur à la légende, les tribulations d'une force aveugle », dans J. Roux (dir.), Rumeurs et renommées dans la Bible, Paris, Honoré Champion, 2021.

\section{NOTES}

1. Selon Platon, Épiméthée ayant procédé à la distribution des qualités aux êtres vivants, il apparaît que « les autres animaux [sont] convenablement pourvus sous tous les rapports, tandis que l'homme est tout nu, pas chaussé, dénué de couvertures, désarmé » (Protagoras 322c) ; «L'homme n'est qu'un roseau, le plus faible de la nature [...] : une vapeur, une goutte d'eau suffit pour le tuer » (B. Pascal, Pensées, 1982, p. 212).

2. Nous examinerons le cycle de Samson qui bénéficie d'un texte canonique d'une grande densité littéraire; en revanche nous ne retiendrons pas ici l'affaire du talon d'Achille, dont les traces littéraires, absentes de l'Iliade, apparaissent de manière trop laconiques, notamment dans l'Achiléide de Stace (1971, p. 79).

3. Samson serait naïf selon D. Luciani ("Samson: l'amour rend aveugle», 2009, p. 325) et E. Greenstein («The Riddle of Samson », 1981, p. 245). Il serait même idiot selon E. Assis ("The Structure and Meaning of the Samson Narratives », 2014, p. 7). Pourtant, ces caractérisations contredisent d'autres passages du cycle de Samson.

4. Je me base sur les traductions de la Bible hébraïque par A. Chouraqui (1989) et par le Rabbinat français, que j'adapte le cas échéant.

5. Je développe cette lecture dans «Samson, de la rumeur à la légende, les tribulations d'une force aveugle », 2021.

6. J. et W. Grimm, Contes pour les enfants et la maison, 2009, p. 460.

7. Ibid., p. 459.

8. Si l'on écarte la solution de facilité selon laquelle un conte reposerait sur un univers entièrement invraisemblable. En effet, je considère que le conte merveilleux comporte bien une part d'invraisemblance, plus ou moins grande selon les récits, et parfois limitée au personnel (fée, ogre, diable, etc.), à quelques objets magiques et aux conditions initiales, mais il conserve, pour l'essentiel, une vraisemblance des motivations psychologiques, notamment implicites à travers les actions des personnages et sous-entendues dans les silences du texte.

9. P. Delarue et M.-L. Ténèze, Le Conte populaire français, 2002, p. 134. À noter que ce catalogue ne retranscrit qu'un résumé du conte.

10. Ibid.

11. Ibid.

12. Un exemple littéraire savoureux se trouve dans la fable «Les deux Rats, le Renard et l'œuf » (1679) de La Fontaine : «Deux rats [...] trouvèrent un œuf. / [...] Le bien empaqueter, / Puis des pieds de devant ensemble le porter, / Ou le rouler, ou le traîner, / C'était chose impossible autant que hasardeuse. » (Fables, 1948, p. 241).

13. Soit l'énigme suivante: "Un coffre sans charnière, ni serrure, ni couvercle, ni clef, qui pourtant renferme un trésor doré ! » (J. R. R. Tolkien, Le Hobbit, 2012, p. 105).

14. Voir le thème mythique de l'œuf cosmique. 
15. J. Bru, Le Conte populaire français. Contes merveilleux. Supplément au Catalogue de Paul Delarue et Marie-Louise Ténèze, 2017, p. 95. Je souligne.

16. A. Afanassiev, Les Contes populaires russes, 2000, p. 220.

17. Ibid., p. 221.

18. Ibid.

19. Ibid.

20. Ibid.

21. Alors que l'ogre du « Petit Poucet » de C. Perrault, lui, ne s'en laisse pas compter : « [...] voilà donc comme tu veux me tromper [...]. » (Contes, 2012, p. 249).

22. Les Mille et Une Nuits, 2006, p. 123.

23. Ibid.

24. Ibid.

25. Ibid.

26. Ibid., p. 124.

27. Ibid.

28. Ibid.

29. Ibid.

30. Ibid., p. 125.

31. Ibid.

32. Ibid., p. 125-126. Je souligne.

33. Ibid., p. 123-127.

34. Ibid., p. 125.

35. Ibid.

36. Ibid., p. 103.

37. Ibid., p. 108.

38. Face à cette incongruité, la critique reste généralement silencieuse. Robert Alter constate de manière indigente : «Samson a un penchant pour les secrets [...]. » (Ancient Israel, 2013, p. 206, ma traduction de l'anglais).

39. L'épisode de Gaza est souvent considéré comme ayant pour seule fonction de manifester les pulsions sexuelles de Samson (voir D. Luciani, «Samson : l'amour rend aveugle », 2009, p. 324), en omettant la prise en compte de sa nouvelle démonstration de force. En fait, sa soi-disante concupiscence peut être comprise comme une provocation.

\section{RÉSUMÉS}

De nombreux récits mettent en scène des personnages a priori invincibles, de la Bible hébraïque (Samson), aux contes russes d'Afanassiev (Kachtcheï), en passant par les contes populaires (sous la catégorie de Corps-sans-Âme, ATU 302). Dans tous les cas, ces personnages sont finalement vaincus, non par un personnage encore plus fort qu'eux, mais par la divulgation réelle ou supposée de leur secret. Le paradoxe étant que généralement ce sont eux-mêmes qui finissent par le révéler dans une hubris de toute-puissance.

Many tales feature supposedly invincible characters, from the Hebrew Bible (Samson) to Afanassiev Russian tales (Koschei) to folktales (under the type ATU 302 The Giant without a heart). In all the cases, those characters are eventually vanquished, not by someone even stronger than 
they are, but because of the real or alleged disclosure of their secret. Paradoxically, they usually end up revealing it themselves, out of a hubris of omnipotence.

INDEX

Mots-clés : Bible hébraïque, Samson, Contes pour les enfants et la maison, « La boule de cristal » (Grimm), Conte populaire français, « La Belle des Belles» (Afanassiev), Kachtcheï, Les Mille et Une Nuits, secret, invincibilité

Keywords : Hebrew Bible, Samson, Kinder- und Hausmärchen, "The Crystal Ball” (Grimm), French Folk Tales, Alexander Afanasyev, Koschei, Arabian Nights, secret, invincibility 